\title{
Recurrent proptotic diplopia due to congestive expansion of cavernous haemangioma with relapsing right-sided cardiac failure
}

\author{
Denis O’Mahony, Eamon O’Neill
}

\begin{abstract}
Summary
A 75-year-old man with a recent history of pulmonary embolism, presented with collapse followed by a gran mal seizure and right-sided non-pulsatile proptosis. On recovery, he had diplopia on lateral and upward gaze and signs of congestive cardiac failure. Further pulmonary embolism was proven by lung scintigraphy. Computed tomography of his orbits confirmed a contrast-enhancing spaceoccupying lesion of the medial wall of the right orbit, with no intracranial abnormality. The patient was investigated for metastatic tumour as a possible cause of the space-occupying lesion and the unprovoked thromboembolic event, but no evidence of malignancy was found. The orbital lesion was not biopsied because of the risk of bleeding from anticoagulation. Three weeks later, the patient represented with recurrent cardiac failure, proptosis, and diplopia. A transorbital ultrasound confirmed an encapsulated, well-defined vascular lesion, with typical appearances and Doppler flow characteristics of a cavernous haemangioma. Diuretic therapy abolished the proptosis and diplopia in tandem with relief of the cardiac failure. This is the first description of recurrent proptosis with diplopia due to recurrent congestive expansion of an orbital cavernous haemangioma.
\end{abstract}

Keywords: haemangioma; proptosis; diplopia; cardiac failure

Acute unilateral proptosis causing diplopia is usually the result of acute orbital haemorrhage, cavernous sinus thrombosis, or orbital sepsis. It may, however, result from acute congestive expansion of orbital tumours. A resolving, relapsing cause of unilateral proptosis with diplopia occurring in tandem with recurrent right-sided cardiac failure should suggest a vascular lesion communicating with the major veins of the head and neck, such as cavernous haemangioma, as this case illustrates.

\section{Case report}

A 75-year-old man was brought to hospital as an emergency following collapse followed by a gran mal seizure. On admission to the Accident \& Emergency department, he was still uncon- scious but no longer fitting. He had rapid atrial fibrillation (110 beats/min) and marked cyanosis (arterial oxygen saturation $77 \%$ ), moderately elevated blood pressure $(180 / 100 \mathrm{mmHg})$ but no signs of respiratory distress. Signs of chronic lower limb venous insufficiency, a left lower parasternal heave and distended neck veins were noted. There was obvious non-pulsatile proptosis of the right eye without conjunctival suffusion or an overlying bruit. Both the optic disc and fundus looked normal. His neurological status was considered consistent with a post-ictal state. A non-contrast cranial computed tomography (CT) scan was performed in order to exclude intracranial haemorrhage and cavernous sinus thrombosis. The appearances suggested reduced venous drainage from the right orbit but no other abnormalities. A chest $\mathrm{X}$-ray showed cardiac enlargement and a right basal pleural effusion, but no other abnormalities. Later, collateral history from the patient's daughter revealed that he had been hospitalised 2 months previously with an extensive right leg deep venous thrombosis associated with pulmonary embolism (confirmed on lung ventilation/ perfusion scintigraphy) and that he had been taking warfarin since then with doubtful compliance.

The patient was treated with intravenous heparin infusion and $40 \%$ inspired oxygen. Five hours after the ictus, the patient had recovered consciousness, and complained of new-onset painless double vision and dyspnoea at rest. Over the next 4 days, his overall clinical status improved, but he continued to have right-sided proptosis and double vision on extremes of lateral gaze. He remained cyanosed with signs of right-sided cardiac failure. Because of persisting right-sided proptosis, on the fourth hospital day the patient had a further cranial orbital CT scan with intravenous contrast. This showed a well-defined, contrast-enhancing retrobulbar mass on the medial side of the right orbit, medial to the medial rectus muscle, stretching along the ethmoid plate and extending into the retrobulbar space, displacing the medial rectus muscle (figure). The differential diagnosis was tumour mass or haemangioma. Clinical, laboratory and radiological investigation for extraorbital malignancy proved negative, and the patient was referred for a clinical ophthalmological assessment. Arising from this, an orbital duplex ultrasound confirmed an ovoid vascular tumour $(21 \times 18 \times 12 \mathrm{~mm})$ with scan appearances and 


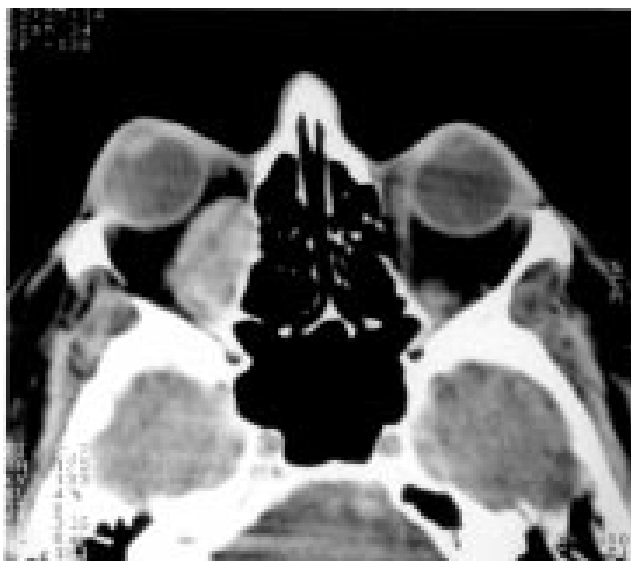

Figure Contrast-enhanced CT scan of the orbits, showing a well-defined ovoid lesion extending along the medial wall of the orbit, adjacent to the ethmoid sinus, and proptosis of the right eye

Doppler blood flow characteristics highly consistent with cavernous haemangioma.

Over the next 2 weeks, the patient continued to improve with warfarin, digoxin and diuretics. As the signs of right heart failure regressed, so did the proptosis, and the diplopia eventually resolved completely. The patient was discharged from hospital 22 days after admission on warfarin, digoxin $125 \mu \mathrm{g}$ daily and bumetanide $1 \mathrm{mg}$ daily. Unfortunately, 3 weeks later, he was readmitted to hospital as an emergency with a 4-day history of relapsing cardiac failure, with recurrent right-sided proptosis and diplopia. Cardiac failure with right-sided pleural effusion was confirmed on chest radiograph. He was treated with intravenous frusemide for 3 days with good effect. Once again, the patient's proptosis receded and the diplopia resolved with stabilisation of his cardiac failure. After treatment of the acute heart failure, an echocardiogram confirmed biventricular systolic dysfunction, bilateral $\mathrm{AV}$ valve regurgitation and a mildly elevated pulmonary artery pressure $(26 \mathrm{mmHg})$.

At out-patients follow-up, it was not possible to reproduce the proptosis and diplopia by means of head-down tilt testing or internal jugular venous obstruction by compression at the root of the neck on the affected side. With supervised drug compliance, the patient's cardiac failure has remained stable and the proptosis has not recurred. This, coupled with the patient's guarded prognosis due to his marked degree of heart failure, has meant that surgery has not been considered necessary.

\section{Discussion}

Right-sided cardiac failure presenting with diplopia due to congestive expansion of a retrobulbar cavernous haemangioma has not been described in the literature previously. Cavernous haemangioma is the most common benign orbital tumour in adults ('cavernous' refers to the macroscopic appearance of the tumour, and does not imply any anatomical association with the cavernous sinus). Typical appearances are those of large ectatic or 'cavernous' spaces, lined by flattened endothelial cells overlying a variable mantle of closely aligned spindle cells. Although cavernous haemangiomas may occur in children, they are generally tumours of adulthood, with most cases occurring between 30 and 60 years of age. From a clinicopathological viewpoint, there is no evidence that cavernous haemangiomas arise from capillary haemangiomas of childhood. They can occur anywhere in the orbital cavity, but most typically in the soft tissues behind the globe, as in this case. The mass is usually sited within the muscle cone, most often inferior and lateral to the optic nerve. Cavernous haemangiomas usually present with slowly progressive unilateral proptosis, and may be associated with optic disc oedema and choroidal folds visible on funduscopy. Visual disability may result from a high degree of relative hypermetropia or from optic disc compression. Features encountered in some other orbital vascular lesions, like intermittent and fluctuating proptosis, postural variation of proptosis and visual distortion, pulsation or bruit, haemorrhagic cyst or phlebolith formation are usually not found with orbital cavernous haemangioma. ${ }^{1}$

Transorbital duplex ultrasound, which demonstrates the well-defined, capsular, septate structure of the tumour and its highly vascular nature, is currently the method of choice for diagnosis. Because of the high degree of definition of the lesion with ultrasound, tumour angiography or venography is now seldom required. Occasionally, however, in cases of suspected difficult surgical access, direct puncture angiography may be necessary to define the extent of the lesion and its relations to surrounding structures, but there are risks of bleeding and trauma. The availability of high resolution ultrasound and contrast CT or magnetic resonance imaging means that direct puncture angiography is no longer undertaken as part of the routine pre-operative assessment of orbital cavernous haemangiomas.

Definitive treatment is surgical resection, since the natural history is generally that of slowly progressive expansion and worsening proptosis with visual symptoms. In the present case, surgery was not considered necessary because of the resolution of the diplopia concurrent with improvement of the patient's cardiac failure, and the fact that the patient has had no further visual acuity or other ophthalmic symptoms after 9 months of follow-up.

Recurrent congestive expansion of orbital cavernous haemangiomas causing proptosis and diplopia has not been described in the literature before. Acutely raised pulmonary artery pressure may be transmitted to the veins of the head and neck, including the facial vein. The facial vein lacks valves, so that raised facial venous pressure may in turn be transmitted to the orbit via its anastomosis with the superior ophthalmic vein, the principle venous drainage channel of the orbit. ${ }^{2}$ This is the probable anatomical basis for the congestive expansion of the cavernous haemangioma in this case. The pressure within the cavernous haemangioma was not measured. However, the close tempo- 
ral association of proptosis/diplopia with the episodes of right-sided cardiac failure and the clearcut resolution of the diplopia simultaneous with resolution of the right heart failure strongly supports a causal link. Contrary to expectation, however, at out-patient follow-up, transient obstruction of venous blood flow by neck root compression did not reproduce proptosis or diplopia in this patient. It may be that sustained, prolonged, elevation of venous pressure (as occurred with the two episodes of right heart failure) is required to alter the compliance characteristics of the tumour sufficient for congestive expansion. In addition, the patient was unable to tolerate neck root compression in the supine posture for more than 2 minutes because of marked orthopnoea. Although fluctuating proptosis is occasionally a feature of cavernous haemangioma, ${ }^{3}$ accentuation of proptosis by venous flow obstruction in the root of the neck is not documented in the literature as a reliable physical sign. Similarly, change in proptosis with change in posture has not been described in a large series of cases. ${ }^{1}$

The cause of the gran mal seizure in association with the initial presentation of this patient's diplopia was not thought to be related to the orbital lesion. The CT scan did not show any evidence of cerebral infarction or other

\footnotetext{
1 Harris GJ, Jakoviec FA. Cavernous hemangioma of the
orbit: a clinicopathologic analysis of sixty-six cases. In: FA Jakoviec, ed, Ocular and adnexal tumors. Birmingham, Alabama, USA: Aesculapius Publishing Company, 1978; pp Alabam
}

\section{Summary points}

- cavernous haemangioma is the most common benign orbital tumour in adults

- the natural history of these tumours is one of slow expansion and gradual onset of symptoms

- rarely, subclinical cavernous haemangiomas may undergo acute congestive expansion with associated acute proptosis and visual distortion, as with acute right heart failure

abnormality that would readily explain the ictal event. The seizure did occur in association with marked systemic hypoxia (due to pulmonary embolism), which lowers seizure threshold. The patient has been hospitalised subsequently on two occasions with seizures. $\mathrm{He}$ has recovered completely on both occasions, without signs of residual neurological deficit.

We are not aware of any reported case of right heart failure presenting with recurrent diplopia due to recurring acute congestive expansion of a cavernous haemangioma. Recurrent congestive expansion of a cavernous haemangioma in association with right-sided cardiac failure should be considered as a cause of recurrent proptosis.

2 Moore KL. Clinically oriented anatomy. Baltimore/London: Williams and Wilkins, 1980; p 975.

3 Reese AB. Tumours of the eye. Hagerstown, Maryland, USA: Harper \& Row, 1976; pp 272.

\title{
Primary lymphoma of the bladder treated successfully with mitozantrone gel
}

\author{
P Porter, A J Cornaby, M Al-Hilali, A M Chakrabarti
}

\author{
Department of \\ Surgery, Dorset \\ County Hospital, \\ Dorchester, Dorset \\ DT1 2JY, UK \\ P Porter \\ A J Cornaby \\ M Al-Hilali \\ A M Chakrabarti \\ Correspondence to \\ P Porter, Specialist Registrar, \\ Airedale General Hospital, \\ Skipton Road, Steeton, \\ Keighley, West Yorkshire \\ BD20 6TD, UK \\ Accepted 19 April 1999
}

\begin{abstract}
Summary
We describe a young man who presented with a short history of painless haematuria. Subsequent investigations and biopsy of lesions found in his bladder at cystoscopy confirmed the diagnosis of primary lymphoma of the bladder. Computed tomography studies confirmed the disease was localised to his bladder. Unfortunately, the tumour was not eradicated by radiotherapy. However, it was successfully treated with intravesical mitozantrone given in a novel gel formation. Three years after diagnosis the patient remains well with no evidence of recurrence.
\end{abstract}

Keywords: lymphoma; bladder; mitozantrone

A 22-year-old man presented with a 4-week history of painless haematuria. He had no other symptoms and was otherwise healthy. He had no significant medical or surgical history and clinical examination was entirely normal. Routine blood tests, urine microscopy and culture, and urine cytology failed to detect any abnormality. Excretory urography was also normal.

He underwent rigid cystoscopy. Two discrete nodules $(1 \mathrm{~cm}$ and $0.5 \mathrm{~cm}$ in diameter) were found in the trigone and base of his bladder and these were resected. Histological examination confirmed them to be small cell, or primary lymphoma of the bladder of the mucosa-associated lymphoid tissue (MALT) type. Computed tomography (CT) of the pelvis, abdomen and thorax confirmed the disease to be restricted to his bladder. Bone marrow aspirate was also normal. The patient underwent radiotherapy (20 courses, total tumour dose 4000 cGy). At cystoscopy 3 
months later there was no obvious evidence of tumour, but random biopsies from the affected area confirmed histologically the presence of lymphoma. He then underwent six cycles of intravesical mitozantrone, prepared by mixing $10 \mathrm{mg}$ of mitozantrone in $15 \mathrm{ml}$ of $\mathrm{KY}-\mathrm{gel}^{\mathrm{TM}}$ (Johnson \& Johnson Ltd, Maidenhead, UK) and administering the mixture into the urethra and bladder via a syringe fitted with a urethral tip. The patient tolerated the treatment well and did not suffer any side-effects.

Subsequent regular cystoscopic examination and biopsy of the bladder base and trigone have failed to detect any signs of recurrent disease. Serial magnetic resonance imaging of the pelvis, abdomen and thorax performed during the follow-up period have also failed to show any signs of lymphoma.

\section{Discussion}

Malignant primary lymphoma of the bladder is a rare condition, accounting for only $0.2 \%$ of all extranodal lymphomas and $0.1 \%$ of primary bladder tumours. ${ }^{1}$ Only a relatively small number of cases have been reported in the world literature.

The disease usually presents in middle-age, most commonly with haematuria. ${ }^{23}$ Recurrent cystitis is a common symptom, but was not present in this patient. Treatment with early radiotherapy seems to give the best outcome; surgical intervention, including radical cystectomy, has no effect on prognosis. ${ }^{4}$ In our patient the tumour persisted or recurred after radiotherapy and an alternative treatment was therefore necessary.

Mitozantrone is used systemically in the treatment of lymphoma and other tumours,

1 Isaacson PG. Malignant lymphoma of the urogenital tract. n: Isaacson PG, Norton A, eds, Extranodal lymphomas, 1st edn. Churchill Livingstone, 1994; pp 285-6.

2 Ohsawa M, Aozasa K, Horiuchi K, Kanamaru A. Malignant lymphoma of bladder. Cancer 1993;72:1969-74.

3 Stewart DJ, Green R, Futter N, et al. Phase I and pharmacology study of intravesical mitoxantrone for recurrent superficial bladder tumours. ₹ Urol 1990;143:714-6.

4 Simpson RHW, Bridger JE, Anthony PP, James KA, Jury I. Malignant lymphoma of the lower urinary tract. Br f Urol 1990;65:254-60.

\section{Summary/learning points}

- painless haematuria should be investigated thoroughly

- primary lymphoma of the bladder is an extremely rare condition but is usually radiosensitive

- intravesical mitozantrone is useful in the treatment of bladder tumours and can be administered in a gel

and is effective in the treatment of recurrent superficial bladder tumours when administered locally. ${ }^{56}$ It is a synthetic anthraquinone with structural similarities to doxorubicin but exhibits a lower side-effect profile. In the treatment of recurrent superficial bladder tumours, a dose of $20 \mathrm{mg}$ is effective and at this dose there is little systemic absorption from the bladder. ${ }^{56}$ We chose the smaller dose of $10 \mathrm{mg}$ because the drug was administered intravesically in a gel formulation, and was thus less likely to be diluted and more likely to remain in contact with the affected area of the bladder for longer. The risk of side-effects with the lower dose was also reduced. The gel formulation is a new concept in the treatment of bladder tumours situated in the region of the bladder base. The drug was easy to administer, did not require catheterisation, and hopefully resulted in longer contact of the drug with the trigone and bladder base.

There were no adverse effects from this treatment. This is the first reported case of mitozantrone used to treat primary lymphoma of the bladder.

5 Tyrrell CJ, Daniel I, Hammonds J, Choa G. The use of mitozantrone in superficial bladder tumours. Proceedings of the 4th UK Mitozantrone Symposium. Birmingham, UK, 1990; pp 121-6.

6 Namasivayam S, Whelan P. Intravesical mitozantrone in recurrent superficial bladder cancer : a phase II study. $B r f$ Urol 1995;75:740-3. 


\title{
High-dose intravenous glucagon in severe tricyclic poisoning
}

\author{
Penelope R Sensky, Stephen A Olczak
}

\begin{abstract}
Summary
A case of dothiepin poisoning complicated by cardiogenic shock is described. Hypotension was resistant to conventional inotropes but responded rapidly to high-dose intravenous glucagon. Glucagon should be considered as a useful therapeutic positive inotrope and a potentially antiarrhythmic agent in severe tricyclic antidepressant overdose.
\end{abstract}

Keywords: tricyclic antidepressants; dothiepin; poisoning; cardiogenic shock; glucagon; inotropic support

Department of General Medicine, Pilgrim Hospital, Boston, Lincs, PE 21 9QS, UK

P R Sensky

S A Olczak

Correspondence to Dr PR Sensky, Department of Cardiology, Glenfield Hospital, Groby Road, Leicester LE3 9QP, UK

Accepted 22 March 1999
Tricyclic antidepressants are widely prescribed for depressive disorders, insomnia and chronic pain conditions. Unfortunately, these agents are also commonly taken in overdose, causing life-threatening arrhythmias, hypotension and seizures. In a significant proportion of cases, hypotension is profound and resistant to conventional therapeutic strategies. Glucagon is a rarely used positive inotrope, and we report its successful use in the treatment of severe cardiogenic shock secondary to dothiepin excess.

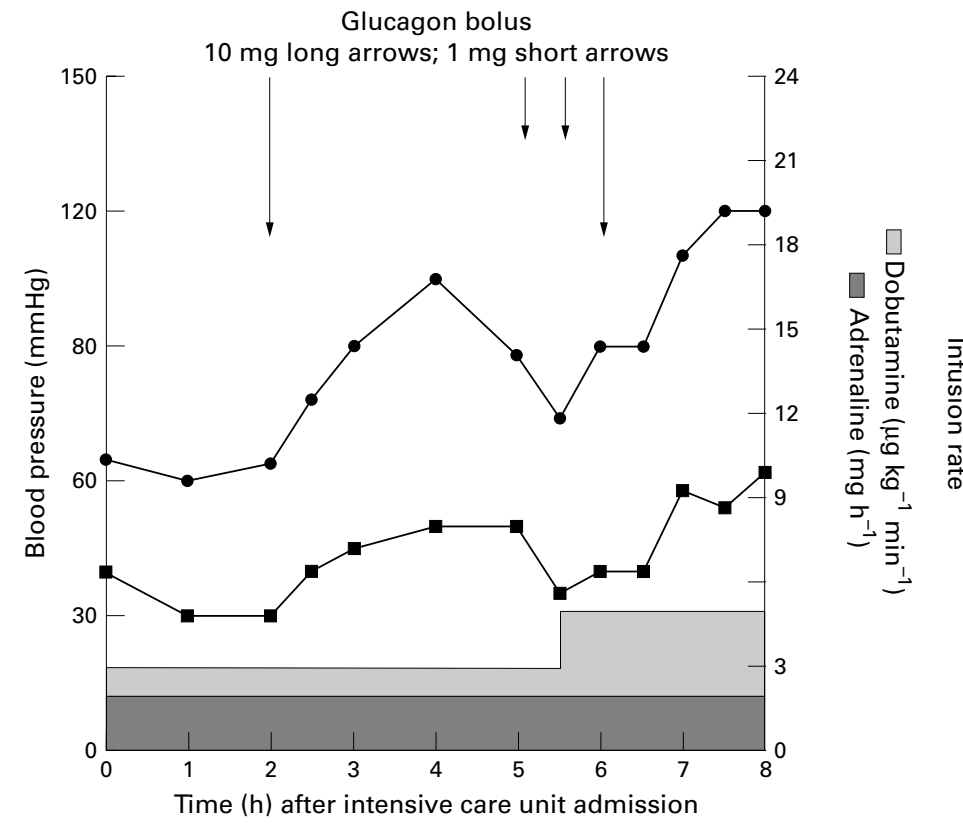

Figure Blood pressure response to inotropic support

\section{Case report}

A 36-year-old woman was brought to casualty unconscious. A drug overdose was suspected and the ambulance crew reported that coproxamol, ibuprofen, antidepressants and alcohol had been present in the house. On examination she had spontaneous respiration, a tachycardia of 120 beats $/ \mathrm{min}$, blood pressure of 60/30 $\mathrm{mmHg}$, dilated pupils, bilateral brisk reflexes, extensor plantar responses and a Glasgow Coma Scale of 7 . Shortly after transfer to the intensive care unit (ICU), a respiratory arrest necessitated intubation and ventilation. Full blood count and renal function were normal, potassium $3.2 \mathrm{mmol} / 1$, INR 1.5 and $\mathrm{pH} 7.29$. Electrocardiogram showed a broad complex tachycardia with QRS width of $0.16-0.2 \mathrm{~s}$, rate 100 beats/min. Colloid and crystalline solutions were given, with an initial brief rise in blood pressure. Charcoal and lactulose were administered via a nasogastric tube and an acetyl-cysteine infusion was commenced. Grand mal seizures were controlled with diazepam and etomidate. Adrenaline, noradrenaline, ephedrine, dobutamine and aminophylline were used in an attempt to correct persisting systolic hypotension of 50-60 $\mathrm{mmHg}$. Two hours after ICU admission, a systolic pressure of $70 \mathrm{mmHg}$ was achieved using infusions of $2 \mathrm{mg} / \mathrm{h}$ adrenaline, and $3 \mu \mathrm{g} /$ $\mathrm{kg} / \mathrm{min}$ dobutamine. On the advice of Guy's Poisons Unit, a 10-mg bolus of intravenous glucagon was given. This was followed by an immediate sustained rise in systolic pressure to over $90 \mathrm{mmHg}$ which persisted for 3 hours (figure) following which she again became profoundly hypotensive. Two further 1-mg boluses of intravenous glucagon were administered with no effect. A further bolus of $10 \mathrm{mg}$ stimulated a rapid increase in blood pressure which was then maintained overnight with infusions of $5 \mu \mathrm{g} / \mathrm{kg} / \mathrm{min}$ dobutamine, $2 \mathrm{mg} / \mathrm{h}$ adrenaline, and $2.5 \mu \mathrm{g} / \mathrm{kg} / \mathrm{min}$ dopamine. One hour after the second 10-mg dose of glucagon she reverted to sinus rhythm. The following day she was awake and responding to commands. Ventilation and inotropic support were weaned off over the next 24 hours. Initially higher mental functions were impaired with short-term memory loss, intermittent confusion and visual hallucinations but by day 5 she had made a full recovery and was transferred to psychiatric care. Admission serum toxicology showed dothiepin: $2.58 \mathrm{mg} / 1$, desmethyldothiepin: $0.51 \mathrm{mg} / \mathrm{l}$, paracetamol: $135 \mathrm{mg} / 1$, diazepam: $0.33 \mathrm{mg} / \mathrm{l}$, nordiazepam: $0.12 \mathrm{mg} / \mathrm{l}$, 
propoxyphene: not detected, confirming that a substantial overdose of a tricyclic antidepressant had been ingested.

\section{Discussion}

Tricyclic antidepressants are still commonly prescribed, despite the advent of newer and safer alternatives. Dothiepin in particular has been associated with high intrinsic toxicity in excess. ${ }^{1}$ Tricyclic poisoning is complicated by profound hypotension, dysrrhythmias, and seizures, and has a high mortality rate. Circulatory shock may be refractory to inotropic support. ${ }^{2}$ Such hypotension is brought about by inhibition of noradrenaline uptake at central presynaptic sites, blockade of peripheral postsynaptic adrenergic receptors and reduced myocardial catecholamine levels. Glucagon has dose-dependent positive inotropic and chronotropic qualities. ${ }^{3}$ It increases myocardial intracellular calcium concentration by stimulating adenyl cyclase, thus enhancing myocardial contractility. This action is thought to occur at nonadrenergic receptors. Thus it is an established treatment for toxicity due to excessive beta-blockade and has been used in calcium channel blocker overdose. ${ }^{4}$ Additional antiarrhythmogenic properties may be due to

1 Buckley NA, Dawson AH, Whyte IM, Henry DA. Greater toxicity in overdose of dothiepin than of other tricyclic antidepressants. Lancet 1994;343:59-62.

2 Buchman AL, Dauer J, Geiderman J. The use of vasoactive agents in the treatment of refractory hypotension seen in tricyclic antidepressant overdose. F Clin Psychopharm 1990;10: 409-13.

\section{Clinical features of tricyclic antidepressant overdose}

- dilated pupils

- respiratory depression

- coma

- hypotension due to arrhythmias, reduced cardiac output, and peripheral vasodilatation - seizures

direct membrane effects. Glucagon has rarely been used in the treatment of severe tricyclic poisoning ${ }^{5}$ but theoretically it is a valuable vasoactive agent as its actions are independent of the adrenoreceptors which are affected in tricyclic excess. In our patient, refractory hypotension secondary to proven dothiepin toxicity persisted despite the use of several standard inotropes. The adjunct of high-dose glucagon appeared to make an important contribution to cardiovascular stabilisation during the resuscitation period. We therefore suggest that the use of glucagon at these dosages should be further evaluated in this situation and more frequently considered for use in cases of severe hypotension and arrhythmias secondary to tricyclic antidepressant toxicity.

3 Farah AE. Glucagon and the circulation. Pharmacol Rev 1983;35:181-217.

4 Doyon S, Roberts J. The use of glucagon in a calcium channel blocker overdose. Ann Emerg Med 1993;22:1229-33.

5 Sener EK, Gabe S, Henry JA. Response to glucagon in imipramine overdose. Clin Tox 1995;33:51-3. 


\title{
A variant of pyothorax-associated lymphoma
}

\author{
C Cheung, M Schonell, A Manoharan
}

\begin{abstract}
Summary
A case of pleural lymphoma that developed after an episode of empyema is described. This may be a variant presentation of the rare yet distinct condition termed pyothorax-associated lymphoma. This condition was first recognised in Japan; there have been only a few reports in Western countries to date. A feature of this case is the relatively short interval between diagnosis of empyema and subsequent development of lymphoma.
\end{abstract}

Keywords: lymphoma; pyothorax-associated lymphoma; empyema

The entity 'pyothorax-associated lymphoma' was first reported by Aozasa in 1987, after reviewing 37 cases of pleural lymphoma in Japan. The presentation of this special subtype of lymphoma was characteristically preceded by 20-30 years of pleural irritation (pyothorax /artificial pneumothorax for tuberculosis treatment). ${ }^{1}$ There have been sporadic reports of this condition since, with the majority of cases described being immunoblastic or large cell types. ${ }^{2} 3$ The current case describes the development of pleural lymphoma within a short time frame after diagnosis and surgical drainage of an empyema.

\section{Case report}

A 70-year-old Caucasian man presented to the St George Hospital in December 1996, with a one-month history of increasing shortness of breath and left pleuritic chest pain. Further history revealed a weight loss of $3 \mathrm{~kg}$, anorexia, and night sweats. The chest X-ray showed a large left pleural effusion. Percutaneous aspiration of the effusion yielded only $15 \mathrm{ml}$ of white turbid fluid. Computed tomography (CT) of the chest showed a large loculated fluid collection occupying most of the left hemithorax.

The patient's history included mild heredi-

St George Hospital, Kogarah, NSW 2217, Australia Department of Clinical Haematology

C Cheung

A Manoharan

Department of

Respiratory Medicine M Schonell

Correspondence to Prof A Manoharan

Accepted 19 April 1999 operation, a thick-walled empyema with 800 $\mathrm{ml}$ of pus was evacuated. Postoperative recov- ery was uneventful. Routine microscopy and cultures for bacteria and acid-fast bacilli were unable to identify a causal organism.

Over the subsequent few months the patient was followed up as an out-patient. Persistent left lower lobe atelectasis prompted two bronchoscopic examinations. On each occasion, a slit-like narrowing of the apical segmental bronchus of the left lower lobe was noted. Bronchial biopsy showed inflammatory changes, including lymphoid infiltrates. Bronchial washings were negative for acid-fast bacilli and malignant cells.

At follow-up in December 1997, the patient was well and routine haematology and biochemistry tests were normal. The chest X-ray and thoracic CT scan, however, showed several right-sided pleural-based mass lesions of variable size, the largest measuring $3 \times 2 \mathrm{~cm}$ adjacent to the right heart border. One of these lesions had extended into the spinal canal in the upper thoracic region. There was also a mass lesion in the right mid zone with several smaller nodules in the right lung. On the left side, there was chronic collapse of the lower lobe, and pleural thickening at the lung base, consistent with a previous empyema drainage. Fine needle aspiration biopsy of one of the right-sided pleural lesions yielded atypical lymphoid cells, of mixed small and large cell types. While highly suspicious of lymphoma, the findings were insufficient for diagnosis.

A repeat CT scan, including the abdomen as part of the staging process, confirmed the soft tissue masses on the right chest wall and collapse of the left lower lobe. No mediastinal or axillary lymphadenopathy was present. Homogenous splenomegaly was noted, consistent with the history of hereditary spherocytosis. There was no other abnormality in the abdomen. A second fine needle pleural biopsy showed a population of large malignant lymphoid cells on cytology. Immunocytochemistry confirmed B cell monoclonality.

Further investigations included a bone marrow biopsy, which showed no evidence of lymphomatous infiltration. A gallium scan showed gallium avid disease confined within the thorax, which correlated with the lymphoma deposits visible on the CT scans.

The patient was commenced on combination chemotherapy, consisting of chlorambucil, vincristine, procarbazine, and prednisone (LOPP), which was tolerated very well. After five treatment cycles given on a monthly basis, the restaging CT scan demonstrated resolution of the right pleural masses, and repeat gallium scan confirmed this improvement. At the time 
of this report, the patient is in clinical remission from lymphoma.

\section{Discussion}

The majority of cases of pyothorax-associated lymphoma (PAL) described in the literature occurred in Japan; there have been less than 10 cases reported in Western countries. ${ }^{2}$ Environmental factors such as Epstein-Barr virus exposure, ${ }^{3}$ or other as yet undetermined factors, may be a crucial trigger to malignant transformation, thus explaining the geographical variation in incidence. One relatively constant feature of PAL appears to be the presence of inflammation or irritation of the pleura prior to development of lymphoma. Tuberculosis, or artificial pneumothorax used for treatment thereof, is a common culprit. ${ }^{1}$ Association with Epstein-Barr virus genome within tumour cells has been noted. ${ }^{1-3}$ Several authors have been able to demonstrate unusual cytokine concentrations in the lymphoma-induced pleural effusion fluids. Interleukins 6 and 10 have been implicated. ${ }^{4}$ Cytokine stimulation exerted on local cells may be the key to de-differentiation of normal reactive lymphocytes. In addition, high frequencies of $\mathrm{p} 53$ mutations have also been found in lymphoma cells of this type. ${ }^{15}$

In the present case, there was no history of exposure to tuberculosis, and examination of the empyema fluid for $M$ tuberculosis was negative. The causal organism of the empyema eluded identification. The radiotherapy that

1 Aozasa K. Pyothorax-associated lymphoma. Int $\mathcal{f}$ Haematol 1996;65:9-16.

2 Ascani S, Piccioli M, Poggi S, et al. Pyothorax-associated ymphoma: description of the first two cases detected in Italy. Ann Oncol 1997;8:1133-8.

3 Ohsawa M, Tomita Y, Kanno H, et al. Role of Epstein-Barr virus in pleural lymphomagenesis. Mod Pathol 1995;8:84553.

4 Danbara M, Takano Y, Fujino Y, Okayasu I, Shionoya S. Development of pyothorax-associated pleural lymphoma in

\section{Learning points}

- a pleural lymphoma developing subsequent to local inflammation may belong to a distinct subtype called 'pyothorax-associated lymphoma'

- cytokines associated with the inflammatory process have been implicated in the malignant transformation

- such a lymphoma is responsive to chemotherapy, and remission can be achieved

the patient received for carcinoma of the bladder was limited to the pelvic region, and probably has no bearing on the chest pathology.

While the bulk of the lymphoma was in the right hemithorax, the initial site of disease was probably at the slit-like narrowing of the left lower lobe segmental bronchus. This lesion predated the other right-sided pleural masses, and showed atypical lymphoid infiltrates on bronchial biopsy. Empyema-induced local inflammation in the left lower lobe could, as postulated in the literature, have led to dedifferentiation and malignant transformation of reactive lymphocytes.

The fact that the lymphoma was the second malignancy in this patient, may imply some form of predisposition towards malignancy development, be it inherited or acquired. In turn, this could explain the unusually short interval between the episode of empyema and the subsequent development of lymphoma.

relation to focal cytokinetic condition and Epstein-Barr virus infection. Acta Haematol 1998;99:41-4.

5 Hongyo T, Kurooka M, Taniguchi E, et al. Frequent p53 mutations at dipyrimidine sites in patients with pyothoraxassociated lymphoma. Cancer Res 1998;58:1105-7.

6 Kanno H, Naka N, Yasunaga Y, et al. Production of the immunosuppressive cytokine Interleukin-10 by EpsteinBarr virus expressing pyothorax-associated lymphoma. Am f Pathol 1997;150:349-57. 


\section{Oral presentation of an oesophageal mucosal tear}

\author{
Sandeep Uppal, P R De
}

\section{Summary}

Tears of the oesophageal wall following sudden forceful vomiting are well documented in literature. In Boerhaave's syndrome there is transmural rupture associated with complications including pneumothorax, pneumomediastinum, surgical emphysema and shock. In Mallory-Weiss syndrome mucosal tears are associated with haematemesis and shock. In neither of these conditions has intraluminal obstruction been described as an aetiological factor. We present a case with similar pathophysiology where oesophageal obstruction by a meat bolus followed by forceful vomiting led to an oesophageal mucosal tear and presentation of a band of oesophageal mucosa in the oral cavity. The patient did not develop any complications and made an uneventful recovery following conservative management.

Keywords: Boerhaave's syndrome; oesophageal wall rupture; vomiting; Mallory-Weiss syndrome

Acute damage to the wall of the oesophagus may be due to spontaneous tears, foreign body ingestion, instrumentation, penetrating injuries and blunt trauma. These tears may be limited to the mucosa or they may be transmural. Presentation includes chest pain, abdominal pain, haematemesis, surgical emphysema, pneumomediastinum, pneumothorax and shock. The morbidity and mortality associated with this condition is significant and early recognition and prompt management is vital for a favourable outcome.

Department of Otolaryngology, Queen's Hospital, Burton Hospitals NHS Trust, Belvedere Road, Burton upon Trent, Staffordshire DE13 0RB, UK S Uppal P R De

Correspondence to Mr PR De

Accepted 20 April 1999

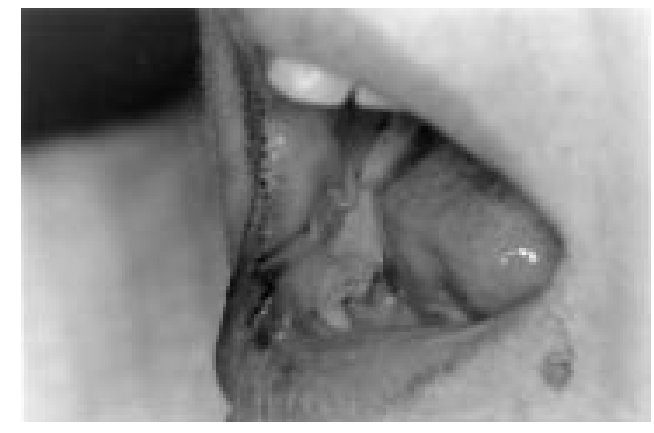

Figure 1 Photograph showing the oesophageal mucosa lying on the right side of the tongue and protruding out of the oral cavity

\section{Case report}

A 43-year-old woman presented with a onehour history of dysphagia following ingestion of a meat bolus. Soon after presentation she vomited producing the meat bolus, $60 \mathrm{ml}$ of fresh blood and a broad band of mucosa which protruded out of the oral cavity. The patient complained of severe pain at the root of neck. There was no epigastric or interscapular pain, but there was some tenderness at the root of the neck. The patient had no signs of shock, surgical emphysema or stridor. A thick band of mucosa was seen lying alongside the right side of the tongue (figure 1). This was traced to the post-cricoid area on indirect laryngoscopy. A minimal traction on the mucosa (to rule out the possibility of a free-lying mucosal band) caused excruciating pain at the root of the neck. This suggested its attachment to the post-cricoid area/upper part of oesophagus.

Preparations were made for an emergency oesophagoscopy to assess the situation and to excise the mucosal band lest it should cause acute airway obstruction. On her way to the theatre the patient consecutively swallowed, regurgitated and eventually swallowed the mucosal band. Under anaesthesia the oral cavity, oropharynx and hypopharynx were normal. The post-cricoid area and the upper oesophagus had irregular oedematous mucosa. A circumferential mucosal tear was identified $30 \mathrm{~cm}$ from the incisors. The oesophagus was found to contain fresh blood but normal mucosa below this level. The band of mucosa could not be identified, having reverted to its normal position from the post-cricoid area to the tear at $30 \mathrm{~cm}$. A nasogastric tube was passed.

The patient's condition remained stable postoperatively. Intravenous amoxycillin $1 \mathrm{~g}$ and clavulanic acid $200 \mathrm{mg}$ were started. She was kept nil by mouth and fed by the nasogastric tube. Gastrograffin swallow performed on the fourth postoperative day showed irregularity of the mucosa in the upper third of the oesophagus but did not reveal any perforation

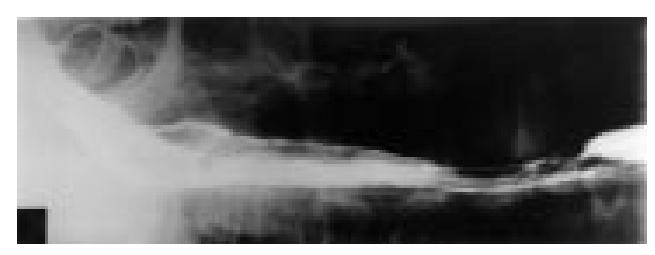

Figure 2 Gastrograffin swallow performed on the fourth post-operative day showing irregularity of the mucosa in the upper third of the oesophagus. There is no evidence of transmural oesophageal rupture 
(figure 2). The patient made an uneventful recovery and was asymptomatic at review 4 weeks later. Fibre-optic oesophagogastroscopy at this time showed a small hiatus hernia and changes of oesophagitis in the lower part of the oesophagus. The mucosa in the upper twothirds of the oesophagus was normal.

\section{Discussion}

Two types of oesophageal damage are well documented after sudden forceful vomiting. In Boerhaave's syndrome, a tear occurs through all the layers of the left lateral wall of the oesophagus just above the diaphragm, produced by sudden increase in oesophageal pressure. The term is generally reserved for spontaneous rupture without intraluminal or extraluminal trauma. ${ }^{1}$ In the act of vomiting the diaphragm and the abdominal muscles contract violently upon the dilated stomach and force gastric contents into the oesophagus. When the oesophagus is open, passage out of the mouth is assured but when there is an obstruction at higher levels in the gastrointestinal tract, oesophageal pressure rises and gastric contents burst through the wall of the lower end of oesophagus. The rupture is usually sharp and linear and penetrates the entire wall of the oesophagus. The initial symptom reported is severe pain in chest, back or abdomen $(83 \%)$ followed by excessive vomiting $(19 \%)$, haematemesis $(1.7 \%)$, dyspnoea $(38 \%)$ and shock $(32 \%){ }^{2}$ There may be pain on swallowing and hoarseness. In $40 \%$ patients, spontaneous rupture of the oesophagus is preceded by a history of heavy drinking. ${ }^{2}$ Peptic ulcer disease is present in $41 \%$, neurological disease in $10 \%$, and $5 \%$ of patients are healthy. ${ }^{2}$ Later the patient may develop subcutaneous emphysema of the neck and pleural effusion. Radiological examination in the early stages shows air confined to mediastinum; later pneumothorax, pleural effusion and hydropneumothorax may occur. Contrast studies with Hypaque or barium may reveal perforations.

Another condition of a similar nature is Mallory-Weiss syndrome. In this condition small linear tears are found in the mucosa of

1 Spiro HM. Clinical gastroenterology, 4th edn. New York: McGraw Hill, Inc, 1993.

2 Brauer RB, Liebermann-Meffert D, Stein HJ, Bartels H, Stewart JR. Boerhaave's syndrome; analysis of literature and report of 18 new cases. Dis Esophagus 1997;10:64-8.

3 Harris JM, Di Palma JA. Clinical significance of Mallory Weiss tears. Am f Gastroenterol 1993;88:2056-8.

\section{Learning points}

- sudden forceful vomiting may lead to either Mallory-Weiss syndrome or Boerhaave's syndrome

- in Mallory-Weiss syndrome, small linear tears are found in the mucosa of oesophageal wall and gastro-oesophageal junction. Endoscopy is the method of diagnosis

- the gastro-intestinal haemorrhage of Mallory-Weiss lesion is associated with regurgitation, vomiting, increased abdominal pressure, excessive alcohol consumption and portal hypertension. Haemorrhage may be excessive, leading to shock

the oesophageal wall or gastro-oesophageal junction. ${ }^{1-3}$ This condition is more common in patients with hiatus hernia and because of milder vomiting the tear is limited to the mucosa without involving the muscular layers. ${ }^{1}$ The gastrointestinal haemorrhage of MalloryWeiss lesion is associated with regurgitation, vomiting, increased abdominal pressure, excessive alcohol consumption and portal hypertension. ${ }^{3}$ The amount of bleeding may vary from a small amount to massive amounts causing shock and requiring transfusions. Endoscopy is the optimum method of diagnosis. ${ }^{4}$ Endoscopic findings include peptic ulcers, gastritis, duodenitis, varices and hiatus hernia. ${ }^{3}$ Barium studies are usually unhelpful. ${ }^{1}$

We present an unusual case of mucosal tear of the oesophagus which does not conform to the above-mentioned entities but has similar pathophysiology. It can be suggested that it amounts to an extreme form of Mallory-Weiss syndrome. It is likely that the sudden increase in pressure and forceful expulsion of the foreign body caused a oesophageal mucosal tear at $30 \mathrm{~cm}$ from the incisors. The mucosa of the upper part of the oesophagus was dissected off the muscle and carried into the oral cavity. However, the mucosa remained attached to the post-cricoid area. We were unable to find any other report of this unusual presentation ${ }^{5}$ and feel that this is probably the first such account.

4 Grassi R, Romano L, Diettrich A, Rossi G, Pinto A. Inkomplettes Boerhaave-Syndrom der zervikalen spieserohre.
Incomplete Boerehaave syndrome of the cervical esopha[Incomplete Boerehaave syndrome
gus] Aktuelle Radiol 1995;5:360-2.

gus] Aktuelle Radiol 1995;5:360-2.
5 Pate JW, Walker WA, Hammond Coli Jr. F, Owen W, Johnson WH. Spontaneous rupture of esophagus: a 30-year experience. Ann Thorac Surg 1989;47:689-92. 


\title{
Follicular carcinoma in a functioning struma ovarii
}

\author{
A Bhansali, V Jain, A Rajwanshi, S Lodha, R J Dash
}

\begin{abstract}
Summary
We describe a case of follicular carcinoma in a functioning struma ovarii, which presented as an ovarian mass in a patient who had undergone a near-total thyroidectomy for a benign lesion. She underwent bilateral salpingo-oophorectomy and received radiotherapy and L-thyroxine treatment with no evidence of metastases in 4 years follow-up.
\end{abstract}

Keywords: struma ovarii; follicular carcinoma

Struma ovarii is an uncommon tumour, and its malignant transformation is rare. ${ }^{1}$ In most instances, diagnosis of malignant struma ovarii is made postoperatively. ${ }^{2}$ We report a patient who had undergone near-total thyroidectomy many years earlier for haemorrhage into a thyroid nodule, who presented with an ovarian mass which was diagnosed as a functioning struma with follicular carcinoma, recognised during hormone replacement therapy (HRT).

\section{Case report}

A 47-year-old woman presented with an euthyroid solitary nodule in June 1987. The nodule was hypoechoeic with poor ${ }^{131}$ I-uptake on scan and had features of follicular neoplasm on fine needle aspiration cytology. Her serum $\mathrm{T}_{3}$ and $\mathrm{T}_{4}$ were normal $(1 \mathrm{ng} / \mathrm{ml}$ and 100 $\mathrm{ng} / \mathrm{ml}$, respectively). She had a near-total thyroidectomy for haemorrhage into the nodule. However, the detailed histology of the nodule excluded malignancy. She had received $150 \mu \mathrm{g}$ L-thyroxine daily until May 1990. Since then she had remained euthyroid with no palpable goitre and normal serum $\mathrm{T}_{3}$ and $\mathrm{T}_{4}$

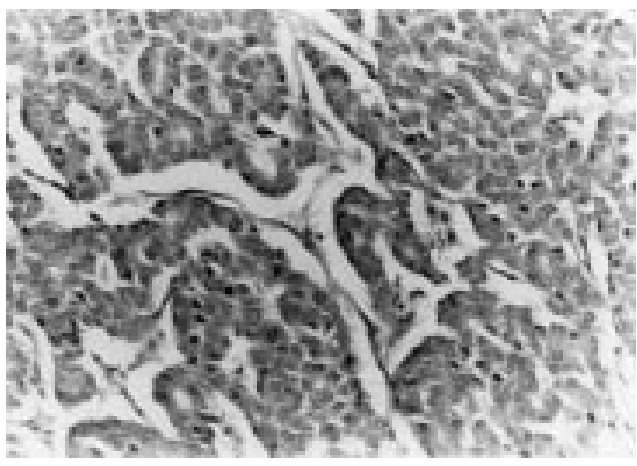

Figure 1 The section shows sheets of thyroid follicular cells with thin colloid $(\mathrm{H} \& \mathrm{E}$, orig $\times 256)$ levels. In March 1994, she complained of irregular cycles and experienced hot flushes. She was put on HRT with conjugated oestrogen and medroxyprogesterone after excluding gynaecologic abnormality by vaginal PAP smear and pelvic ultrasound.

Her hot flushes decreased, but after 6 months she had intermenstrual bleeding. A tender right fornix and $5 \times 6 \mathrm{~cm}$ firm mobile mass was found on per vaginal examination and confirmed by pelvic ultrasound. At laparotomy, a $7 \times 6 \mathrm{~cm}$ right ovarian mass was seen with variegated appearance and multiple solid and cystic areas with an intact surface. There was ascites with no visible peritoneal seedlings. Bilateral salpingo-oophorectomy and hysterectomy were performed. Ascitic fluid was negative for malignant cells. Histopathology revealed near-complete transformation of ovarian tissue into sheets of cells arranged in a follicular pattern with thin colloid separated by fibrocollagenous tissue. The follicular cells showed nuclear pleomorphism, mitotic activity and were invading its capsule; there was no vascular invasion. These findings were consistent with a diagnosis of follicular carcinoma in struma ovarii. No other teratomatous elements were seen (figures 1 and 2). Oestrogen and progesterone receptor status by peroxidase anti-peroxidase immunochemistry after microwave retrieval were found to be negative. The left ovary was normal. An ${ }^{131} \mathrm{I}$ whole body scan after 6 weeks of laparotomy revealed uniform uptake in residual thyroid tissue $(2.4 \%$ at $24 \mathrm{~h})$ and no tracer uptake elsewhere. Her unsuppressed serum thyroglobulin level was 4.5 $\mathrm{ng} / \mathrm{ml}$ (normal $<10 \mathrm{ng} / \mathrm{ml}$ ). She had low serum $\mathrm{T}_{3}$ and $\mathrm{T}_{4}(0.55$ and $40 \mathrm{ng} / \mathrm{ml}$, respectively) and an elevated thyroid-stimulating hormone

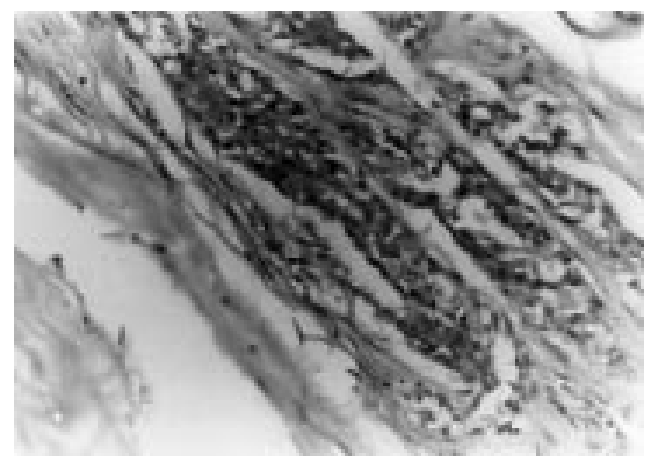

Figure 2 The cancerous cells invading into the capsular lymphatics $(\mathrm{H} \& \mathrm{E}$, orig $\times 256)$ 
(TSH) of $40.8 \mu \mathrm{U} / \mathrm{ml}$, indicating hypothyroidism. She received 4500 rads external beam radiotherapy to the pelvic region in view of the capsular invasion and ascites, together with L-thyroxine therapy.

She is currently well with no evidence of metastases on total body scan at 4 years post-operative follow-up

\section{Discussion}

'Struma ovarii' is a slow-growing ovarian neoplasm, with thyroid tissue as its only or the predominant $(>50 \%)$ constituent. ${ }^{3}$ The ovarian thyroid is histologically and functionally identical to cervical thyroid. ${ }^{4}$ The tumour is a highly specialised subclass of benign cystic teratoma ${ }^{1} ; 95 \%$ of them remain benign while the remainder undergo malignant transformation, with peak frequency during the fifth decade of life. The left ovary is more frequently involved than the right and in $6 \%$ of instances struma ovarii are bilateral. ${ }^{1}$

Struma ovarii often present with abdominal mass, lower abdominal pain, ascites and, uncommonly, hyperthyroidism $(5 \%){ }^{3}$ Past or concomitant thyroid enlargement has been described with struma ovarii $(18 \%)$ which may cause difficulty in its diagnosis. ${ }^{3}$ Retrospectively, the fact that this patient remained euthyroid without L-thyroxine replacement for 4 years, could have given a clue to the presence of a functioning struma ovarii. This was further substantiated when the patient became hypothyroid (TSH $40.8 \mu \mathrm{U} / \mathrm{ml}$ ) promptly after the removal of the ovarian mass. An ${ }^{131} \mathrm{I}$ whole body scan prior to exploratory laparotomy would have picked up the lesion.

Cellular atypia, nuclear pleomorphism, vascular and/or capsular invasion and distant metastases are definite clues to malignant transformation of struma ovarii. ${ }^{4}$ Nuclear pleomorphism, mitotic activity and capsular invasion in our patient suggested the malignant

1 Rosenblum NG, Livolsi VA, Edmonds PR, Mikuta JJ. Malignant struma ovarii. Gynecol Oncol 1989;32:224-7.

2 Gould SF, Lopez RL, Speers WC. Malignant struma ovarii: A case report and literature review. $\mathcal{F}$ Reprod Med 1983;28:415-9.

3 Kempers RD, Dockerty MB, Hoffmann DL, Bartholomew LG. Struma ovarii. Ascitic, hyperthyroid and asymptomatic syndromes. Ann Intern Med 1970;72:883-93.

4 Devaney K, Snyder R, Norris HJ, Tavassoli FA. Proliferative and histologically malignant struma ovarii: a clinicopathologic study of 54 cases. Int f Gynecol Pathol 1993;12:333-43.

\section{Learning points}

- struma ovarii is an uncommon cause of hyperthyroidism

- toxicosis or euthyroidism in a patient with near-total thyroidectomy should raise the suspicion of functioning struma ovarii

- an ${ }^{131} \mathrm{I}$ whole body scan prior to exploratory laparotomy would have established the diagnosis of struma ovarii

- treatment modalities include surgery and/or radioiodine ablation and $\mathrm{L}$-thyroxine in TSH-suppressive doses

nature of the tumour. Capsular invasion carries a poor prognosis. ${ }^{45}$ The majority of malignant struma present as follicular carcinoma, while papillary, anaplastic, and Hurthle cell carcinoma have also been described. ${ }^{5}$

Recognition of an ovarian mass as a struma ovarii during the course of oestrogenprogesterone therapy is an interesting event. Oestrogen modulating TSH secretion and/or TSH exerting a positive influence on follicular growth might have been responsible for the rapid growth of the struma ovarii. ${ }^{6}$ It is noteworthy that the lack of demonstration of oestrogen and progesterone receptors in our case can be explained by the complete transformation of ovarian tissue into struma, as normal thyroid follicular cells do not express oestrogen receptors.

Therapeutic modalities include total abdominal hysterectomy with bilateral salpingooophorectomy, thyroid ablation (radioiodine or surgery) followed by thyroxine-suppressive treatment. Chemotherapy and radiotherapy have been used for recurrent metastatic struma ovarii and anaplastic carcinoma which do not concentrate radioiodine. ${ }^{17}$

5 O'Connell MEA, Fisher C, Harmer CL. Malignant struma ovarii: Presentsation and management. Br f Radiol 1990;63: 360-3.

6 Morley JE. Endocrine control of thyrotoropin secretion. Endocr Rev 1981;2:396-436.

7 Willemse PHB, Oosterhuis JW, Aalders JG, et al. Malignant struma ovarii treated by ovariectomy, thyroidectomy and 131I administration. Cancer 1987;60:178-82. 